\title{
Traditional Chinese medicine Qili qiangxin inhibits cardiomyocyte apoptosis in rats following myocardial infarction
}

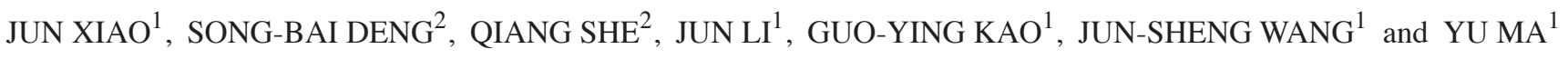 \\ ${ }^{1}$ Department of Cardiology, Chongqing Medical Emergency Center, Chongqing 400014; ${ }^{2}$ Department of Cardiology, \\ The Second Affiliated Hospital of Chongqing University of Medical Sciences, Chongqing 400010, P.R. China
}

Received July 28, 2015; Accepted September 9, 2015

DOI: $10.3892 /$ etm.2015.2759

\begin{abstract}
The aim of the present study was to examine the effect of the traditional Chinese medicine Qili qiangxin on cardiomyocyte apoptosis following myocardial infarction (MI) in a rat model. MI was induced in rats by ligation of the anterior descending coronary artery. Survivors were randomly divided into the sham operation, MI, and Qili qiangxin groups ( $4 \mathrm{~g} / \mathrm{kg}$ per day). After 28 days, infarction size was measured. In the non-infarcted zones (NIZ), the apoptotic index (AI) was measured by terminal deoxynucleotidyl transferase (TdT)-mediated digoxigenin-conjugated dUTP nick-end labeling (TUNEL). Expression of Fas was detected by immunohistochemistry, and the expression of xanthine oxidase (XO) and caspase- 3 by western blot analysis. In addition, the XO and $\mathrm{O}_{2}^{-}, \mathrm{OH}$-scavenging activity of myocardial tissue in NIZ was measured by colorimetry. Compared to the MI group, AI and the expression of Fas and caspase-3 were significantly decreased in NIZ. The activity of XO was also considerably reduced while $\mathrm{O}_{2}{ }^{-}$and $\mathrm{OH}$-scavenging activity was significantly increased in the Qili qiangxin group. Ventricular remodeling was attenuated but there were no significant differences in infarct size (IS) or XO expression levels between the Qili qiangxin and MI groups. In conclusion, the results suggest that Qili qiangxin may inhibit cardiomyocyte apoptosis in NIZ
\end{abstract}

Correspondence to: Dr Yu Ma, Department of Cardiology, Chongqing Medical Emergency Center, 1 Jiankang Road, Yuzhong, Chongqing 400014, P.R. China

E-mail: yr7964@sina.com

Abbreviations: MI, myocardial infarction; ROS, reactive oxygen species; XO, xanthine oxidase; LVEDD, left ventricular end-diastolic dimension; FS, factional shortening; EF, ejection faction; IS, infarct size; TUNEL, terminal deoxynucleotidyl transferase (TdT)-mediated digoxigenin-conjugated dUTP nick-end labeling; DAB, 3,3'-diaminobenzidine; AI, apoptotic index; IOD, integrated optical density; SDS-PAGE, sodium dodecyl sulfate polyacrylamide gel electrophoresis; FADD, Fas-associated death domain protein

Key words: Qili qiangxin, xanthine oxidase, reactive oxygen species, apoptosis, myocardial infarction in rats. The potential mechanism involved may be associated with its ability to reduce reactive oxygen species (ROS) and to depress the expression of Fas and caspase-3.

\section{Introduction}

Cardiomyocyte apoptosis is important in left ventricular (LV) remodeling and dysfunction following myocardial infarction (MI) (1-3), which induces symptomatic heart failure. The large amount of reactive oxygen species (ROS) produced in the ischemic myocardium can induce cardiomyocyte apoptosis $(3,4)$. Accumulating evidence suggests that there is an upregulated expression and activity of xanthine oxidase (XO), which mediates myocardial dilatation by increased production of catalytic ROS (5-7).

The Qili qiangxin capsule was developed according to the theory on main and collateral channels in traditional Chinese medicine, which consisted of extraction or powder from 11 components (8). As a medicine for chronic heart failure, Qili qiangxin was approved by the State Food and Drug Administration of China in 2009 (9,10). Previous studies indicated that the major active ingredients of Qili qiangxin, such as Ginseng, Radix Astragali and Salvia miltiorrhiza, may significantly inhibit the enzymatic activity of XO (11-13) and decrease the production of ROS (13-15).

However, the effect of Qili qiangxin on cardiomyocyte apoptosis remains to be determined. Therefore, the aim of the current study was to investigate the effect of Qili qiangxin on cardiomyocyte apoptosis in rats after MI, as well as its potential mechanism of action.

\section{Materials and methods}

Components and preparation of Qili qiangxin. Qili qiangxin powder was provided by Shijiazhuang Yiling Pharmaceutical Co., Ltd (Shijiazhuang, Hebei, China), and comprises Ginseng, Radix Astragali, Salvia miltiorrhiza, Aconite root, Semen Lepidii Apetali, Cortex Periplocae Sepii Radicis, Rhizoma Alismatis, Carthamus tinctorius, Polygonatum odorati, Seasoned Orange Peel and Ramulus Ginnamomi. The herbal drugs were authenticated and standardized on marker compounds according to the Chinese Pharmacopoeia 2010. The drug powder was dissolved in normal saline at the concentration of $2.67 \mathrm{~g} / \mathrm{ml}(10)$. 
Rat models and experimental protocol. MI was induced in 50 adult male Sprague-Dawley rats weighing 180-230 g (supplied by the Experimental Animal Centre of the Third Affiliated Hospital of the Third Military Medical University, Chongqing, China), by permanent ligation of the left anterior descending coronary artery, as previously described (10). Briefly, the rats were anesthetized (pentobarbital sodium; $30 \mathrm{mg} / \mathrm{kg}$; i.p.), intubated, and mechanically ventilated with a rodent ventilator (TKR-200; Jiangxi Teli Anesthesia Ventilator Co., Ltd, Jiangxi, China). Following thoracotomy on the left chest, the heart was exposed. A superficial suture line (7-0) was passed around the proximal left coronary artery and the suture was tied, and successful occlusion was confirmed by visual cyanosis. The left chest was closed in three layers (ribs, muscles, and skin), 15 min after occlusion, and the rats were allowed to recover from anesthesia on their own. Thirty-one rats survived the operation and were randomly divided into the MI group $(n=16)$ and the Qili qiangxin group (4 $\mathrm{g} / \mathrm{kg}$ per day by gavage, $n=15)$. Five rats were subjected to the same surgical procedure, except for the ligation of the coronary artery, and served as the sham group. Administration of Qili qiangxin commenced $24 \mathrm{~h}$ after the operation, and continued for 28 successive days. The rat experiments were approved by the local institutional animal research committee.

Echocardiographic measurements. Transthoracic Doppler echocardiographic experiments were performed with a commercially available echocardiographic system (GE Vivid 7; GE Healthcare, Fairfield, Connecticut, USA) equipped with a $13 \mathrm{MHz}$ transducer. Briefly, a two-dimensional short-axis view of the left ventricle was obtained at the level of the papillary muscle, in order to record M-mode tracing. The average of left ventricular end-diastolic dimension (LVEDD), factional shortening (FS), as well as ejection faction (EF) were calculated, on the basis of three successive cardiac cycles. The observer was blind to the experimental group assignment.

Measurement of infarct size. After 28 days of surgery, all the rats were weighed and sacrificed by cervical dislocation and the hearts were immediately removed. Hearts of rats were removed and the atria, vasculature and right ventricles were dissected and removed. Infarct size (IS) was determined as previously described (7). Briefly, the sections of the left ventricle were immersed in fixative solution, dehydrated and then embedded in paraffin. Histological sections $5 \mu \mathrm{m}$ were subsequently obtained and stained with hematoxylin and eosin. Endocardial and epicardial circumferences of the infarcted tissue and the left ventricle were determined using image analysis software (Image Pro Plus 4.5; Media Cybernetics, Inc., Silver Spring, MD, USA). IS was calculated as: (endocardial + epicardial circumference of the infarcted tissue)/(endocardial + epicardial circumference of the left ventricle) and expressed as a percentage. The remaining myocardial tissue was frozen in liquid nitrogen and stored at $-80^{\circ} \mathrm{C}$.

Measurement of $\mathrm{O}_{2}^{-} \cdot \mathrm{OH}$-scavenging and $\mathrm{XO}$ activity by colorimetry. High $\mathrm{O}_{2}^{-}$and $\mathrm{OH}$-scavenging activity, and low $\mathrm{O}_{2}{ }^{-}$and $\mathrm{OH}$ levels are usually observed in the myocardium. As per the manufacturer's instructions for the Superoxide Anion Free Radical Detection kit, Hydroxyl Free Radical Detection kit, and XO Detection kit (Nanjing Jiancheng Bioengineering Institute, Nanjing, China), $100 \mathrm{mg}$ of the frozen myocardial tissue was produced into a $10 \%$ homogenate, and the supernatant was removed and mixed with appropriate reagents. When the reaction was finished, absorbance was detected with a 752 Ultraviolet Spectrometer (Qinghua Tech-Apparatus Company, Beijing, China), to calculate the $\mathrm{O}_{2}{ }^{-}$and $\mathrm{OH}$-scavenging activity, as well as $\mathrm{XO}$ activity.

In situ nick end-labeling. Apoptotic nuclei were detected by in situ terminal deoxynucleotidyl transferase (TdT)-mediated digoxigenin-conjugated dUTP nick end-labeling (TUNEL), using an In Situ Cell Apoptosis Detection kit (MK1020; Boster Biotechnology Co., Ltd., Wuhan, China). The sections were processed according to the manufacturer's instructions, with modifications. Briefly, the sections were incubated for $10 \mathrm{~min}$ and quenched in $3 \% \mathrm{H}_{2} \mathrm{O}_{2}$ buffer blocked with endogenous peroxidase, followed by a 10 -min proteolytic digestion in pre-diluted proteinase K (Sigma, St Louis, MO, USA)-exposed antigen binding sites. The slides were then incubated in equilibration buffer for $2 \mathrm{~min}$, followed by incubation in working strength TdT enzyme at $37^{\circ} \mathrm{C}$ for $2 \mathrm{~h}$. The TdT working strength enzyme comprised TdT enzyme and digoxigenin-11-dUTP for extension of the 3'-OH ends of double-or single-stranded DNA of fragmented DNA. This process was followed by stop/wash and anti-digoxigenin-peroxidase steps, which were performed according to the manufacturer's instructions. Color was developed using 3,3'-diaminobenzidine (DAB), which generated a brown reaction product. Positive controls were prepared by treating selected slides with $0.5 \mathrm{mg} / \mathrm{ml}$ DNase I for $10 \mathrm{~min}$ at room temperature. dUTP labelling was not observed when TdT was omitted from the reaction. The number of apoptotic cardiomyocytes and their percentage of total cardiomyocytes were counted under a light microscope. For each slide, five fields were randomly selected, and by using a defined rectangular field area at a magnification of $\mathrm{x} 200$, a total of 200 cells per field were counted. The apoptotic index (AI) was determined as the number of apoptotic cardiomyocytes divided by the total number of cardiomyocytes counted $\mathrm{x} 100 \%$, from a total of 15 fields per heart, with assays performed in a blinded manner.

Agarose gel electrophoresis of DNA. To detect the internucleosomal cleavage of genomic DNA, a hallmark of apoptotic cell death, DNA was isolated from LV tissue and subjected to ethidium bromide $(0.4 \mathrm{mg} / \mathrm{l})$ agarose gel $(1.5 \%)$ electrophoresis.

Immunohistochemical analysis of Fas. The streptavidinbiotin complex (SABC) immunohistochemical technique was used to detect Fas using an SABC-POD kit (SA2002; Boster Biotechnology Co., Ltd). Samples were fixed within 5 min of excision in $10 \%$ neutral buffered formalin and embedded in paraffin blocks. After deparaffinization and rehydration, the samples underwent 10 -min incubation in $3 \% \mathrm{H}_{2} \mathrm{O}_{2}$ in buffer blocked with endogenous peroxidase. The slides were treated with $0.01 \mathrm{M}$ target retrieval solution (natrium citricum buffer; Beijing Leagene Biotechnology Co., Ltd., Beijing, China) at $95^{\circ} \mathrm{C}$ for $10 \mathrm{~min}$ as per the manufacturer's instructions. Non-specific binding was blocked using goat serum in PBS buffer (Boster Biotechnology Co., Ltd.) for 

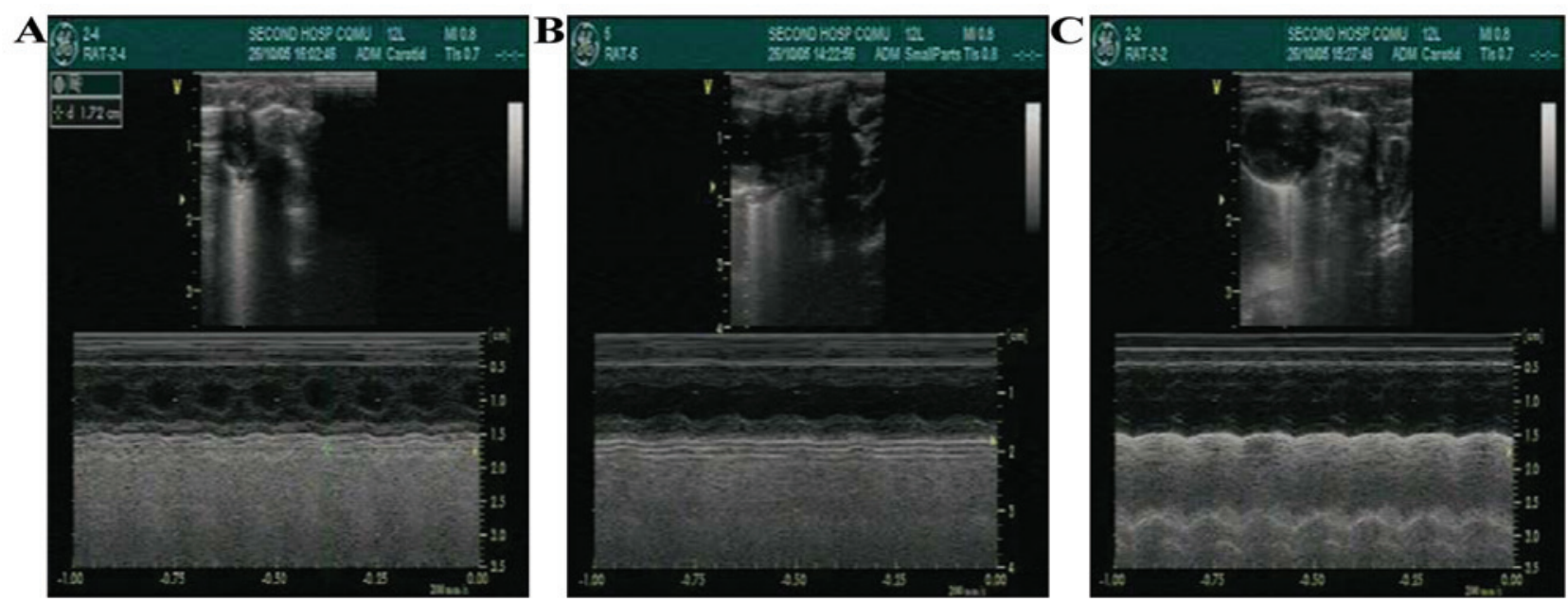

Figure 1. Representative M-mode echocardiograms of the left ventricle in the parasternal short-axis view of 28 days post-myocardial infarction (MI): (A) sham group; (B) MI group and (C) Qiliqiangxin group.

20 min. Primary antibody for rat Fas (sc-7886; 1:400; Santa Cruz Biotechnology, Inc., Santa Cruz, CA, USA) was applied overnight at $4^{\circ} \mathrm{C}$. Following incubation with the initial primary antibody, the slides were placed in biotinylated secondary anti-rabbit (goat absorbed) polyclonal antibody (BA1003; 1:1200; Boster Biotechnology Co., Ltd.) for $20 \mathrm{~min}$. This antibody complex was then detected after 20 min incubation in a streptavidin-biotin-horseradish peroxidase complex. Color was developed using DAB, which generated a brown reaction product. The slides were counterstained with hsematoxylin. Control slides included isotype-matched host-specific antibodies at a dilution of 1:100,10\% primary antibody host-serum, and single (no primary antibody) and double (no primary or secondary antibody) negative controls. For analysis of the positive expression of Fas, the slides were evaluated and Fas cytoplasm staining was quantified from five non-consecutive tissue sections at a magnification of x200 in 10 random fields in a blinded manner. The integrated optical density (IOD) of the positively expressed area was measured as a semiquantitative parameter with image analysis software (Image Pro Plus 4.5).

Western blot analysis of XO and caspase-3. For all the groups, equal amounts $(40 \mu \mathrm{g})$ of protein extracts were loaded and separated, according to their size, by sodium dodecyl sulfate polyacrylamide gel electrophoresis (SDS-PAGE; Boster Biotechnology Co., Ltd.) using 10\% acrylamide gradient. Separated proteins were transferred electrophoretically to a polyvinylidene difluoride membrane (Boster Biotechnology Co., Ltd.). Non-specific sites were blocked by incubation of the membrane in blocking buffer (5\% non-fat dry milk in T-TBS) for $2 \mathrm{~h}$. The membranes were incubated with the indicated primary antibodies [XO: (1:400),sc-20991; SantaCruz Biotechnology, Inc.; caspase-3: (1:400), CPP32; NeoMarkers, Fremont, CA, USA; $\beta$-actin: (1:200); Boster Biotechnology Co., Ltd)] overnight at $4^{\circ} \mathrm{C}$. Horseradish peroxidase-conjugated anti-rabbit immunoglobulin IgG (1:1500; Boster Biotechnology Co., Ltd) was used as a secondary antibody and incubated for $2 \mathrm{~h}$ at $37^{\circ} \mathrm{C}$. Immunoreactive bands were visualized by DAB and photographed. Absorbance analysis of the images was performed using image analysis software (Quantity One 4.4.0).
The densities of $\mathrm{XO}$ and caspase- 3 in relation to $\beta$-actin were, respectively, expressed as $\mathrm{XO} / \beta$-actin and caspase-3/ $\beta$-actin.

Statistical analysis. Data are presented as mean \pm SEM. The parameters were compared using one-way ANOVA, followed in case of significance, by a two-sided Tukey's test for multiple comparisons. $\mathrm{P}<0.05$ was considered statistically significant.

\section{Results}

Echocardiography and infarct size. After 28 days, 6 rats in the MI group and 1 rat in the Qili qiangxin group died. The rats in the sham group survived. Compared to the sham group, LVEDD was increased and FS and EF were significantly decreased in the MI group $(\mathrm{P}<0.01)$. However, compared to the MI group LVEDD was decreased, and FS and EF were significantly increased in the Qili qiangxin group $(\mathrm{P}<0.01)$. Although IS was smaller in the Qili qiangxin group than that in the MI group, the difference was not statistically significant $(\mathrm{P}>0.05)$, (Table I, Figs. 1 and 2). No infarct was detected in the sham group since none of the rats underwent ligation of the coronary artery.

Cardiomyocyte AI. The apoptotic cardiomyocytes were mainly distributed in the border zones of the infarction area (Fig. 3). The AI was markedly higher $(\mathrm{P}<0.01)$ in the MI and Qili qiangxin groups, when compared to the sham group (Table I). However, the AI in the Qili qiangxin group was significantly lower than that in the MI group $(\mathrm{P}<0.01)$.

DNA ladder. Following agarose gel electrophoresis, the myocardial tissue DNA of the sham group exhibited a late band close to the sample well, which was recognized as the normal band pattern for cardiomyocyte DNA. The distribution of DNA fragments in the MI group was dispersed and a typical DNA ladder was observed while the relatively minor dispersion of the DNA fragments was detected in the Qili qiangxin group which made the DNA ladder disappear (Fig. 4).

Fas expression. Fas expression was located on the membrane or in the cytoplasm of the cardiomyocytes (Fig. 5). Compared 
Table I. Effect of Qili qiangxin on ventricular remodeling, cardiomyocyte apoptosis and oxidative stress (mean \pm SD).

\begin{tabular}{|c|c|c|c|}
\hline Group & Sham & MI & Qili qiangxin \\
\hline No. & 5 & 10 & 14 \\
\hline LVEDD (mm) & $5.8 \pm 0.9$ & $7.2 \pm 0.8^{\mathrm{a}}$ & $6.2 \pm 0.5^{\mathrm{b}}$ \\
\hline $\mathrm{FS}(\%)$ & $53.94 \pm 8.41$ & $31.97 \pm 5.88^{\mathrm{a}}$ & $46.50 \pm 7.82^{\mathrm{b}}$ \\
\hline $\mathrm{EF}(\%)$ & $74.86 \pm 5.66$ & $35.47 \pm 2.25^{\mathrm{a}}$ & $56.38 \pm 5.45^{\mathrm{a}, \mathrm{b}}$ \\
\hline IS $(\%)$ & & $30.6 \pm 3.2$ & $28.4 \pm 2.9$ \\
\hline $\mathrm{AI}(\%)$ & $0.5 \pm 0.2$ & $21.1 \pm 1.4^{\mathrm{a}}$ & $6.2 \pm 0.8^{\mathrm{a}, \mathrm{b}}$ \\
\hline Fas (IOD) & $10681 \pm 2079$ & $77,328 \pm 5,151^{\mathrm{a}}$ & $31,925 \pm 3,263^{\mathrm{a}, \mathrm{b}}$ \\
\hline Caspase- $3 / \beta$-actin & $0.04 \pm 0.01$ & $0.45 \pm 0.08^{\mathrm{a}}$ & $0.12 \pm 0.04^{\mathrm{c}, \mathrm{b}}$ \\
\hline $\mathrm{XO} / \beta$-actin & $0.15 \pm 0.06$ & $0.53 \pm 0.09^{\mathrm{a}}$ & $0.51 \pm 0.07^{\mathrm{a}}$ \\
\hline Activity of XO (u/mgpro) & $0.21 \pm 0.05$ & $1.62 \pm 0.19^{\mathrm{a}}$ & $0.43 \pm 0.13^{\mathrm{b}}$ \\
\hline Activity of $\mathrm{O}_{2}^{-}$-scavenging (u/gpro) & $151.38 \pm 9.57$ & $64.77 \pm 8.50^{\mathrm{a}}$ & $97.72 \pm 8.99^{\mathrm{a}, \mathrm{b}}$ \\
\hline Activity of $\mathrm{OH}$-scavenging (u/mgpro) & $253.03 \pm 17.39$ & $91.67 \pm 16.21^{\mathrm{a}}$ & $177.02 \pm 9.56^{\mathrm{a}, \mathrm{b}}$ \\
\hline
\end{tabular}

LVEDD, left ventricular end-diastolic dimension; FS, fractional shortening; EF, ejection fraction; IS, infarct size; AI, apoptotic index; IOD, integrated optical density; $\mathrm{XO}$, xanthine oxidase. ${ }^{\mathrm{a}} \mathrm{P}<0.01 \mathrm{vs}$. sham group, ${ }^{\mathrm{b}} \mathrm{P}<0.01 \mathrm{vs}$. MI (myocardial infarction) group, ${ }^{\mathrm{c}} \mathrm{P}<0.05$.
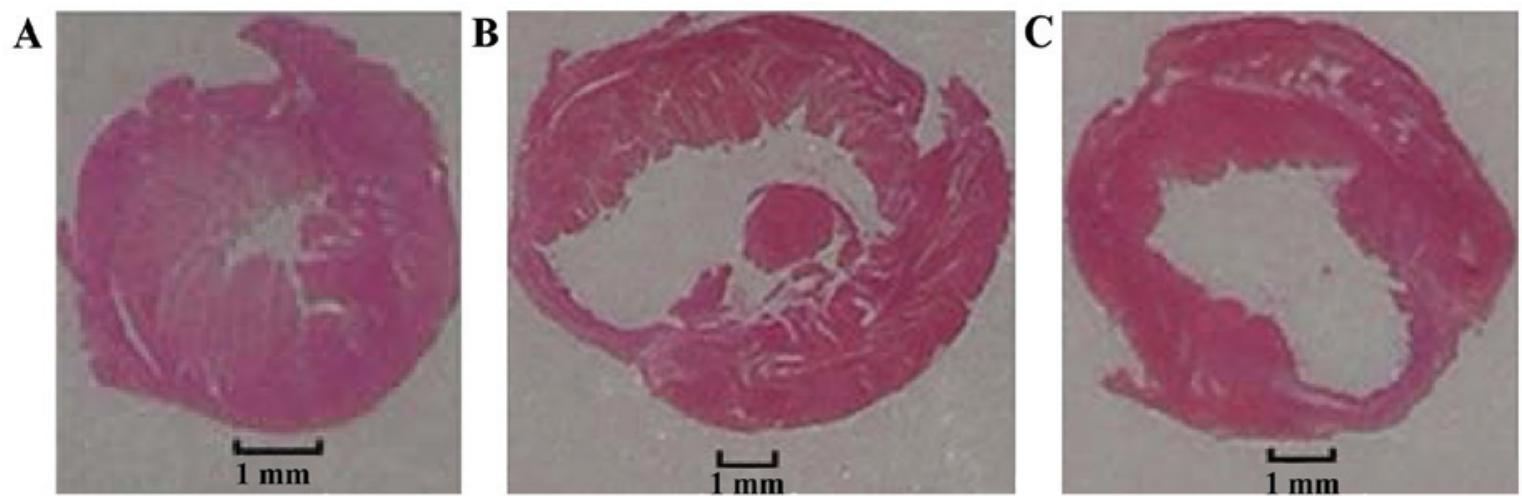

Figure 2. Representative left ventricle cross-section with hematoxylin and eosin staining of 28 days post-myocardial infarction (MI). (A) Sham, (B) MI and (C) Qili qiangxin groups.
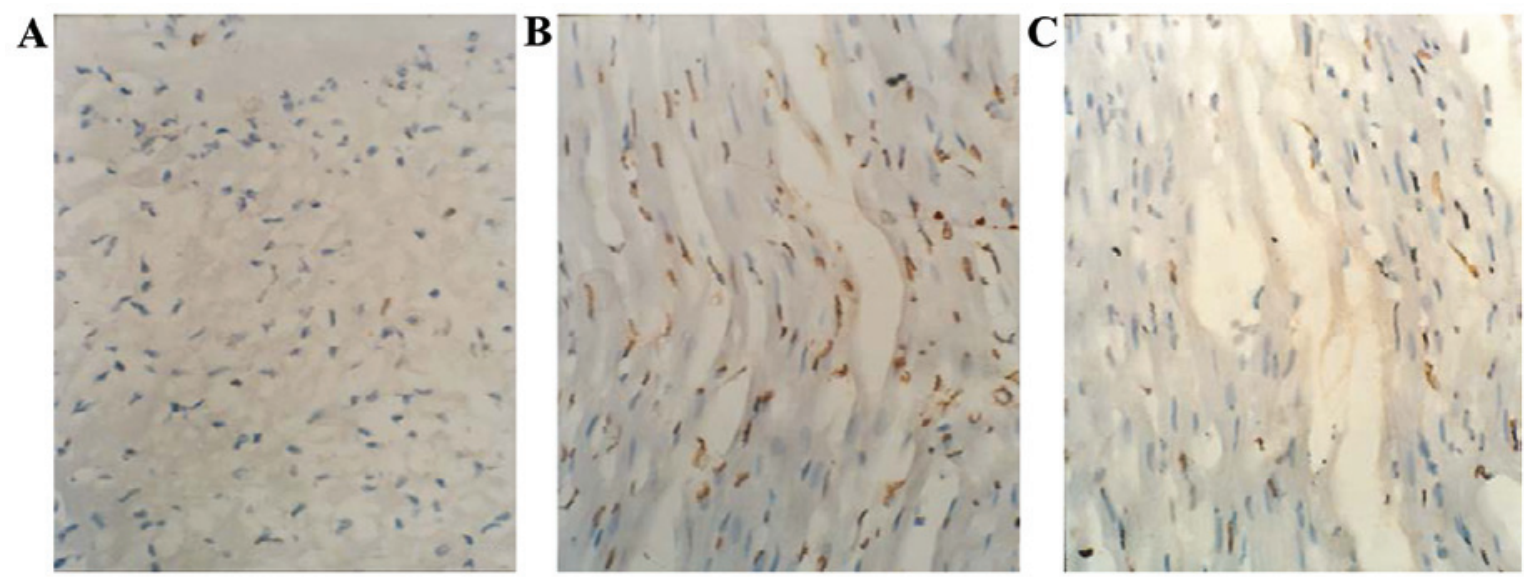

Figure 3. Expression of apoptotic myocardial cells in non-infarcted zones (NIZ) detected by terminal deoxynucleotidyl transferase (TdT)-mediated digoxigenin-conjugated dUTP nick end-labeling (TUNEL) staining (x200). (A) Sham, (B) myocardial infarction and (C) Qili qiangxin groups.

to the sham group, the IOD of Fas was significantly higher in the MI and the Qili qiangxin groups $(\mathrm{P}<0.01)$. However, IOD in the Qili qiangxin group was markedly lower than that in the MI group $(\mathrm{P}<0.01)$ (Table I). 


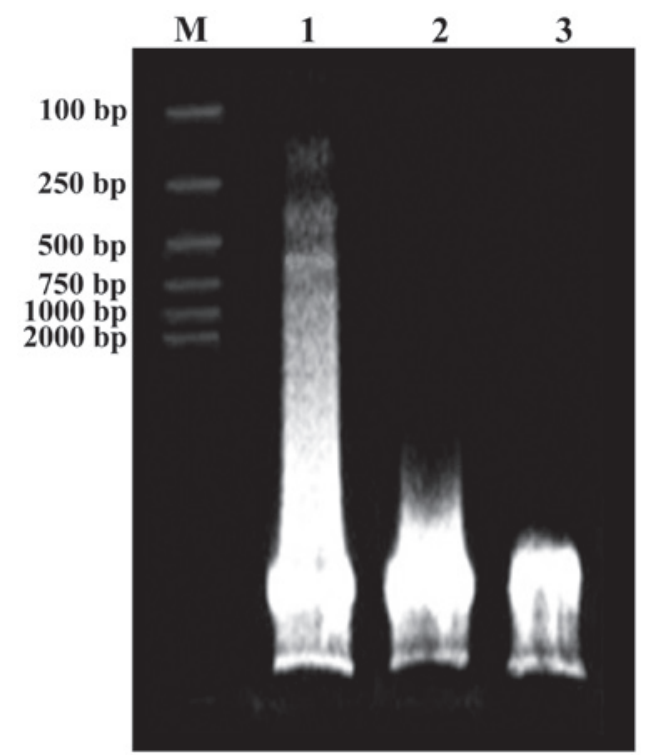

Figure 4. Agarose gel electrophoresis of myocardial deoxyribonucleic acid (DNA) in non-infarcted zones (NIZ): M, DNA marker; 1, myocardial infarction group; 2, Qiliqiangxin group; 3, sham group.

Caspase-3 expression. Caspase-3 expression was similar to that of Fas and was markedly increased in the myocardial tissue of the MI and Qili qiangxin groups $(\mathrm{P}<0.01)$. However, the increase was much lower in the Qili qiangxin group compared to the MI group, although a significant difference was evident between the Qili qiangxin and sham groups $(\mathrm{P}<0.05)$ (Table I and Fig. 6).

Activity and expression of $X O$. Compared to the sham group, $\mathrm{XO}$ activity was significantly increased and the expression was also enhanced in the MI and Qili qiangxin groups $(\mathrm{P}<0.01)$. However, XO activity in the Qili qiangxin group was significantly lower than that in the MI group $(\mathrm{P}<0.01)$, while no significant difference was identified in the expression of $\mathrm{XO}$ between the two groups ( $\mathrm{P}>0.05)$ (Table I and Fig. 6).

$\mathrm{O}_{2}{ }^{-}$and $\mathrm{OH}$-scavenging activity of myocardial tissue. The $\mathrm{O}_{2}-$ and $\mathrm{OH}$-scavenging activity of the myocardial tissue decreased significantly in the MI and Qili qiangxin groups $(\mathrm{P}<0.01$ or $\mathrm{P}<0.05)$. However, the two indicators were significantly higher in the Qili qiangxin group compared to the MI group $(\mathrm{P}<0.05)$ (Table I).

\section{Discussion}

Pharmaceutics of herbal medicine is undergoing rapid development in China. With the progression of modern technology, herbal compound extracts are increasingly being authenticated, standardized, and administered successfully in clinical practice. Qili qiangxin, derived from a group of herbal medicine including Radix Astragali, Ginseng, Salvia miltiorrhiza, Aconite root, and Semen Lepidii Apetali have been used to treat patients with chronic heart failure for two years (8-10). It has been demonstrated that the major active constituents of Qili qiangxin, such as Ginseng, Radix Astragali and Salvia miltiorrhiza, significantly inhibit the enzymatic activity of XO (11-13) and decrease the production of ROS (13-15).
However, whether the improvement of ventricular remodeling by Qili qiangxin is associated with the inhibition of cardiomyocyte apoptosis remains to be determined.

Ventricular remodeling after MI involves expansion of the infarcted area, ventricular dilatation, as well as thinning of the ventricular wall $(16,17)$. Our study was designed to assess whether the administration of Qili qiangxin affected ventricular remodeling. Echocardiography performed after 28 days of surgery showed that LEVDD was decreased while FS and EF were significantly increased in rats that had undergone the MI operation. However, no significant difference was observed for IS between the MI and Qili qiangxin groups. Considering the trend towards a smaller IS in the Qili qiangxin group, this finding may be due to the relatively short duration or small sample size of our study.

Accumulating evidence suggests that a higher level of ROS plays a pathological role through cell signaling pathways, inducing apoptosis (18). ROS is generated intracellularly by activation of nicotinamideadenine dinucleotide phosphate oxidase or XO, uncoupling of nitric oxide synthase, and electron transport and 'leakage' during oxidative phosphorylation in the mitochondria. Biochemical and pharmacological studies suggest that $\mathrm{XO}$ acts as a major source of ROS in the cardiovascular system, and $\mathrm{XO}$-derived ROS has been demonstrated in experimental and clinical heart failure (5-7).

Apoptosis is a distinct type of cell death characterized by a series of typical morphological events, such as shrinkage of the cell, fragmentation into membrane-bound apoptotic bodies and rapid phagocytosis into neighboring cells without the induction of inflammatory response, while the biochemical hallmark of apoptosis is internucleosomal DNA fragmentation. Cardiomyocyte apoptosis has been identified in viable myocardial areas after MI in experimental and human ischemic heart failure, and was regarded as an important factor leading to ventricular remodeling and dysfunction (1-4). As an independent factor, apoptosis in the expansion of the infarcted area is thought to lead to the partial ventricular wall becoming thinner and cavity dilation, i.e., an earlier remodeling (19). By contrast, apoptosis in the border and remote zones of the infarction is the main factor leading to the $\mathrm{LV}$ remodeling and dysfunction in the advanced stage (20).

The Fas death pathway is critical for cardiomyocyte apoptosis, which is easily activated by oxidative stress (21). Fas ligand, an integral membrane protein binding to a Fas trimer can induce a conformational change in Fas that enables its cytoplasmic tail to recruit Fas-associated death domain protein (FADD) through interactions involving death domains in the two molecules. FADD, in turn recruits procaspase- 8 through homotypic interactions involving death effector motifs. The approximation of procaspase- 8 stimulates its autoactivation, followed by the activation of downstream caspase-3 by caspase- 8 which induces apoptosis (22). Zhu et al reported that the conformity between the degree of Fas expression and the phase of an increase in apoptosis indicated that the change in the expression of Fas was closely associated with cardiomyocyte apoptosis (2). Caspase-3 has been confirmed as a dominant executor in the Fas death pathway, a key protease in apoptosis, which can result in DNA degradation and apoptosis by activating CAD (23). Thus inhibition of the activity or function of caspase- 3 may 

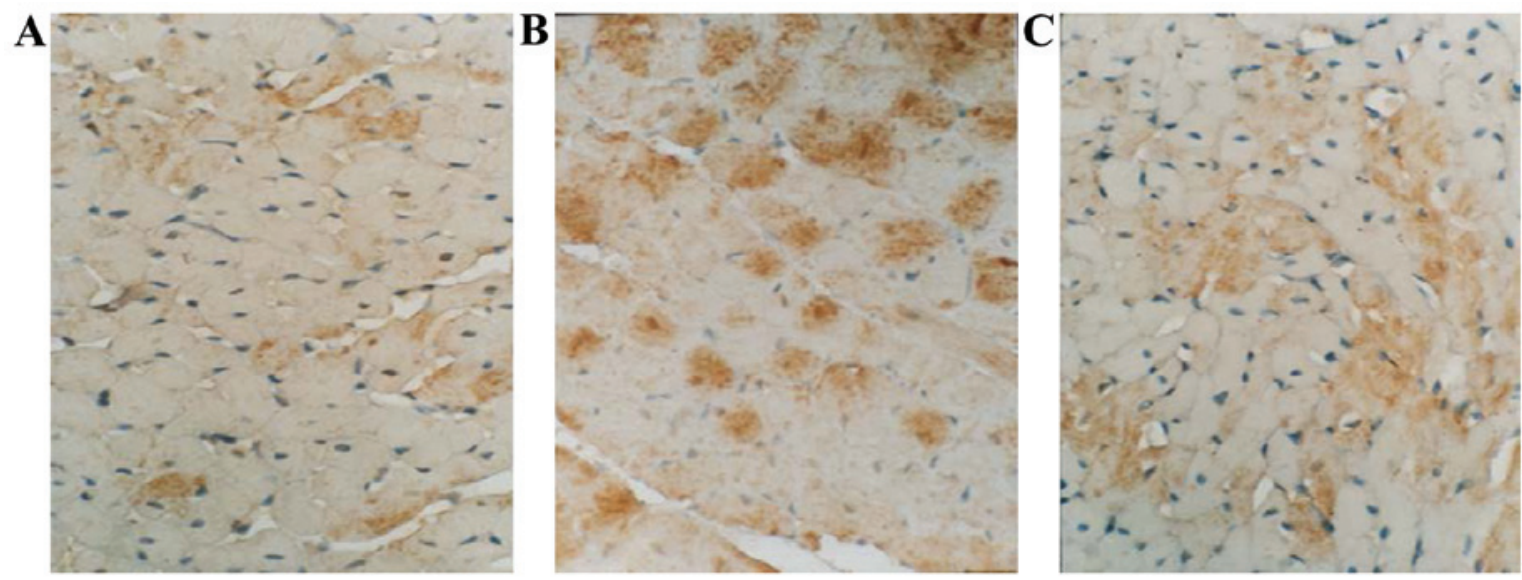

Figure 5. Expression of Fas in non-infarcted zones (NIZ) detected by immunohistochemistry (x200). (A) Sham, (B) myocardial infarction (MI) and (C) Qili qiangxin groups.

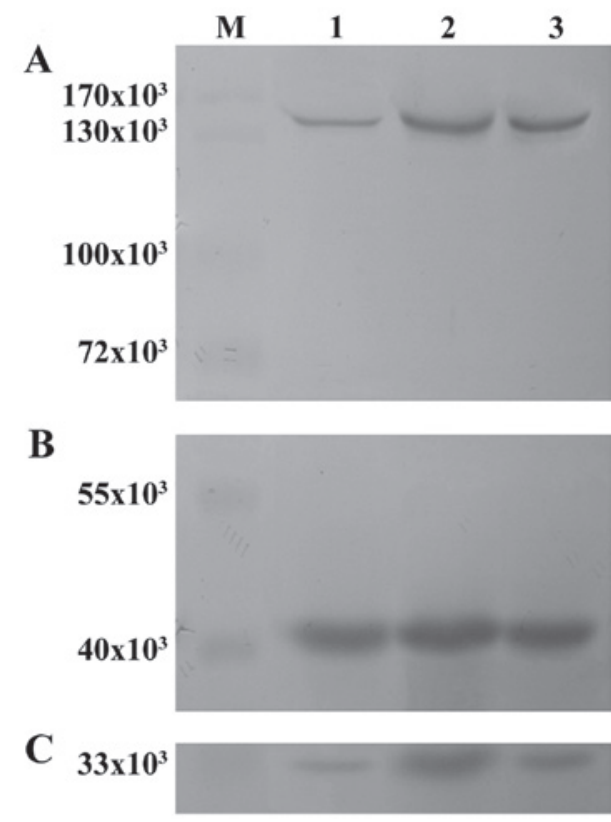

Figure 6. Expression of xanthine oxidase (XO) and caspase-3 in non-infarcted zones (NIZ) detected by western blot analysis. M, protein marker; lane 1 , sham group; lane 2, myocardial infarction group (MI); lane 3, Qili qiangxin group. (A) XO; (B) $\beta$-actin and (C) caspase-3.

depress apoptosis $(24,25)$. Sam et al reported an increase in cell death in the non-infarcted zones (NIZ) after MI along with an increase in caspase-3 activity (20).

Results of the present study suggest that after 28 days of treatment with Qili qiangxin, the AI and expression of Fas and caspase-3 were markedly reduced in the rats that had undergone the MI operation. The DNA ladder, a hallmark indicator of apoptosis, disappeared in these rats, suggesting the anti-apoptotic activity of Qili qiangxin in myocardial tissue in the NIZ.

The potentially cytological and molecular mechanisms by which Qili qiangxin inhibits cardiomyocyte apoptosis remain to be elucidated. These mechanisms may be due to the cumulative or synergistic effects of multiple compounds present in the herbal extract. One possible mechanism is that it relieves apoptosis by reducing LV end-diastolic pressure and mitigates the tension of the ventricular wall (26). For example, Aconite root, a Chinese medicinal herb, has been shown to have positive inotropic, vasodilation and diuretic effects in the management of congestive heart failure (27). Another possible explanation is associated with inhibition of the high production of ROS in the ischemic myocardium. It has been reported that Ginseng, Radix Astragali and Salvia miltiorrhiza, may significantly inhibit the production of ROS (13-15).

ROS $\left(\mathrm{O}_{2}^{-}, \mathrm{OH}\right.$ and $\left.\mathrm{H}_{2} \mathrm{O}_{2}\right)$ which, through multiple intracellular redox signaling pathways $(18,28-30)$, directly damages DNA and mitochondria, promotes the expression of redox sensitive proapoptotic genes (such as Fas, and p53) (31), and activates the inflammatory cytokine-like mitogen-activated protein kinases (32) to manipulate cardiomyocyte apoptosis. It has been previously confirmed that the direct scavenging of $\mathrm{O}_{2}{ }^{-}$or $\mathrm{OH}$ attenuates $\mathrm{LV}$ remodeling and dysfunction $(33,34)$. Glutathione peroxidase, which removes $\mathrm{H}_{2} \mathrm{O}_{2}$ and detoxifies lipid hydroperoxides, is also overexpressed in mouse heart and has been shown to ameliorate post-MI remodeling (35).

$\mathrm{XO}$, a potent enzymatic source of ROS, has an upregulated expression and activity in the ischemic myocardium, while the major active constituents of Qili qiangxin, such as Ginseng, Radix Astragali and Salvia miltiorrhiza may significantly inhibit XO activity (11-13). Results of the present study showed that following administration of Qili qiangxin for 28 days, $\mathrm{XO}$ activity was reduced and $\mathrm{O}_{2}^{-}$, $\mathrm{OH}$-scavenging activity of myocardial tissue was enhanced in the rats that had undergone the MI resection, whereas the expression level of $\mathrm{XO}$ remained the same in the untreated MI group.

In summary, our results in the rats indicate that following administration of Qili qiangxin for 28 successive days, dilation of the left ventricle caused by MI was markedly attenuated. By contrast, in the non-infarct zones XO activity was inhibited and $\mathrm{O}_{2}^{-}, \mathrm{OH}$-scavenging activity of myocardial tissue was enhanced by Qili qiangxin. In addition, the AI, and expression levels of Fas and caspase-3 were also reduced in the same area. The expression level of XO remained the same in the MI group, suggesting that Qili qiangxin acts following the translation process, rather than at the transcription and translation levels. These results confirm our hypothesis that through the combination of XO after MI, Qili qiangxin was able to reduce the 
generation of ROS and inhibit cardiomyocyte apoptosis in the non-infarct zone, and prevent remodeling of the left ventricle.

Qili qiangxin may also inhibit cardiomyocyte apoptosis through other means, for example, by inhibiting the expression of p53 (31), or by reducing the activity of the stress-activated protein kinase (36), or by inhibiting the apoptosis signal-regulating kinase 1 (37). However, these possible mechanisms require further investigation.

\section{Acknowledgements}

This study was partially supported by Chongqing Municipal Health and Family Planning Commission Medical Research Fund, Chongqing, China (20142083).

\section{References}

1. Shah AM and Mann DL: In search of new therapeutic targets and strategies for heart failure: Recent advances in basic science. Lancet 378: 704-712, 2011.

2. Zhu YZ, Zhu YC, Wang ZJ, Lu Q, Lee HS and Unger T: Time-dependent apoptotic development and pro-apoptotic genes expression in rat heart after myocardial infarction. Jpn J Pharmacol 86: 355-358, 2001.

3. Savateev AV and Savateeva-Liubimova TN: Apoptosis-universal mechanisms of cell death and survival in ischemia and reperfusion: Ways to pharmacological control. Eksp Klin Farmakol 73 44-49, 2010 (In Russian).

4. Abbate A, Bussani R, Amin MS, Vetrovec GW and Baldi A: Acute myocardial infarction and heart failure: Role of apoptosis. Int J Biochem Cell Biol 38: 1834-1840, 2006.

5. Gladden JD, Ahmed MI, Litovsky SH, Schiros CG, Lloyd SG, Gupta H, Denney TS Jr, Darley-Usmar V, McGiffin DC and Dell'Italia LJ: Oxidative stress and myocardial remodeling in chronic mitral regurgitation. Am J Med Sci 342: 114-119, 2011.

6. Doehner W and Landmesser U: Xanthine oxidase and uric acid in cardiovascular disease: Clinical impact and therapeutic options. Semin Nephrol 31: 433-440, 2011.

7. Mellin V, Isabelle M, Oudot A, Vergely-Vandriesse C, Monteil C, Di Meglio B, Henry JP, Dautreaux B, Rochette L, Thuillez C, et al: Transient reduction in myocardial free oxygen radical levels is involved in the improved cardiac function and structure after long-term allopurinol treatment initiated in established chronic heart failure. Eur Heart J 26: 1544-1550, 2005.

8. Kang LP, Zhao Y, Yu HS, Liu YX, Xiong CQ, Tan DW, Jia JM, Wang HT, Tian SY and Ma BP: Identification of chemical constituents in qiliqiangxin capsule by UPLC-Q-TOF/MS(E). Yao Xue Xue Bao 46: 1231-1236, 2011 [In Chinese].

9. Liu W, Chen J, Xu T, Tian W,Li Y, Zhang Z and Li W: Qiliqiangxin improves cardiac function in spontaneously hypertensive rats through the inhibition of cardiac chymase. Am J Hypertens 25: 250-260, 2012.

10. Xiao H, Song Y, Li Y, Liao YH and Chen J: Qiliqiangxin regulates the balance between tumor necrosis factor-alpha and interleukin-10 and improves cardiac function in rats with myocardial infarction. Cell Immunol 260: 51-55, 2009.

11. Shen L, Han JZ, Li C, Yue SJ, Liu Y, Qin XQ, Liu HJ and Luo ZQ: Protective effect of ginsenoside Rg1 on glutamate-induced lung injury. Acta Pharmacol Sin 28: 392-397, 2007.

12. Yu DH, Bao YM, Wei CL and An LJ: Studies of chemical constituents and their antioxidant activities from Astragalus mongholicus Bunge. Biomed Environ Sci 18: 297-301, 2005.

13. Liu X, Chen R, Shang Y, Jiao B and Huang C: Superoxide radicals scavenging and xanthine oxidase inhibitory activity of magnesium lithospermate B from Salvia miltiorrhiza. J Enzyme Inhib Med Chem 24: 663-668, 2009.

14. Karmazyn M, Moey M and Gan XT: Therapeutic potential of ginseng in the management of cardiovascular disorders. Drugs 71: 1989-2008, 2011.

15. Chen R, Shao H, Lin S, Zhang JJ and Xu KQ: Treatment with Astragalus membranaceus produces antioxidative effects and attenuates intestinal mucosa injury induced by intestinal ischemia-reperfusion in rats. Am J Chin Med 39: 879-887, 2011 .
16. Konstam MA, Kramer DG, Patel AR, Maron MS and Udelson JE: Left ventricular remodeling in heart failure: Current concepts in clinical significance and assessment. JACC Cardiovasc Imaging 4: 98-108, 2011.

17. Gajarsa JJ and Kloner RA: Left ventricular remodeling in the post-infarction heart: A review of cellular, molecular mechanisms, and therapeutic modalities. Heart Fail Rev 16: 13-21, 2011.

18. Finkel T: Signal transduction by reactive oxygen species. J Cell Biol 194: 7-15, 2011.

19. Abbate A, Biondi-Zoccai GG, Bussani R, Dobrina A, Camilot D, Feroce F, Rossiello R, Baldi F, Silvestri F, Biasucci LM, et al: Increased myocardial apoptosis in patients with unfavorable left ventricular remodeling and early symptomatic post-infarction heart failure. J Am Coll Cardiol 41: 753-760, 2003.

20. Sam F, Sawyer DB, Chang DL, Eberli FR, Ngoy S, Jain M, Amin J, Apstein CS and Colucci WS: Progressive left ventricular remodeling and apoptosis late after myocardial infarction in mouse heart. Am J Physiol Heart Circ Physiol 279: H422-H428, 2000.

21. Lee P, Sata M, Lefer DJ, Factor SM, Walsh K and Kitsis RN: Fas pathway is a critical mediator of cardiac myocyte death and MI during ischemia-reperfusion in vivo. Am J Physiol Heart Circ Physiol 284: H456-H463, 2003.

22. Foo RS, Mani K and Kitsis RN: Death begets failure in the heart. J Clin Invest 115: 565-571, 2005.

23. Schwarz K, Simonis G, Yu X, Wiedemann S and Strasser RH: Apoptosis at a distance: Remote activation of caspase-3 occurs early after myocardial infarction. Mol Cell Biochem 281: 45-54, 2006.

24. Holly TA, Drincic A, Byun Y, Nakamura S, Harris K, Klocke FJ and Cryns VL: Caspase inhibition reduces myocyte cell death induced by myocardial ischemia and reperfusion in vivo. $\mathrm{J} \mathrm{Mol}$ Cell Cardiol 31: 1709-1715, 1999.

25. Saitoh T, Nakajima T, Takahashi T and Kawahara K: Changes in cardiovascular function on treatment of inhibitors of apoptotic signal transduction pathways in left ventricular remodeling after myocardial infarction. Cardiovasc Pathol 15: 130-138, 2006.

26. Hsieh MH and Nguyen HT: Molecular mechanism of apoptosis induced by mechanical forces. Int Rev Cytol 245: 45-90, 2005.

27. Lin JS, Chan CY, Yang C, Wang YH, Chiou HY and Su YC: Zhi-fuzi, a cardiotonic Chinese herb, a new medical treatment choice for portal hypertension? Exp Biol Med (Maywood) 232: 557-564, 2007.

28. Shah AM and Channon KM: Free radicals and redox signalling in cardiovascular disease. Heart 90: 486-487, 2004.

29. Murdoch CE, Zhang M, Cave AC and Shah AM: NADPH oxidase-dependent redox signalling in cardiac hypertrophy, remodelling and failure. Cardiovasc Res 71: 208-215, 2006.

30. Oktyabrsky ON and Smirnova GV: Redox regulation of cellular functions. Biochemistry (Mosc) 72: 132-145, 2007.

31. Li PF, Dietz R and von Harsdorf R: p53 regulates mitochondrial membrane potential through reactive oxygen species and induces cytochrome c-independent apoptosis blocked by Bcl-2 . EMBO J 18: 6027-6036, 1999.

32. McCubrey JA, Lahair MM and Franklin RA: Reactive oxygen species-induced activation of the MAP kinase signaling pathways. Antioxid Redox Signal 8: 1775-1789, 2006.

33. Kinugawa S, Tsutsui H, Hayashidani S, Ide T, Suematsu N, Satoh S, Utsumi H and Takeshita A: Treatment with dimethylthiourea prevents left ventricular remodeling and failure after experimental myocardial infarction in mice: Role of oxidative stress. Circ Res 87: 392-398, 2000.

34. Matsushima S, Ide T, Yamato M, Matsusaka H, Hattori F, Ikeuchi M, Kubota T, Sunagawa K, Hasegawa Y, Kurihara T, et al: Overexpression of mitochondrial peroxiredoxin-3 prevents left ventricular remodeling and failure after myocardial infarction in mice. Circulation 113: 1779-1786, 2006.

35. Shiomi T, Tsutsui H, Matsusaka H, Murakami K, Hayashidani S, Ikeuchi M, Wen J, Kubota T, Utsumi H and Takeshita A: Overexpression of glutathione peroxidase prevents left ventricular remodeling and failure after myocardial infarction in mice. Circulation 109: 544-549, 2004.

36. Verheij M, Bose R, Lin XH, Yao B, Jarvis WD, Grant S, Birrer MJ, Szabo E, Zon LI, Kyriakis JM, et al: Requirement for ceramide-initiated SAPK/JNK signalling in stress-induced apoptosis. Nature 380: 75-79, 1996.

37. Nagai H, Noguchi T, Takeda K and Ichijo H: Pathophysiological roles of ASK1-MAP kinase signaling pathways. J Biochem Mol Biol 40: 1-6, 2007. 